\title{
ORIENTACIONES PARA EL DISEÑO DE ESCENARIOS PARA LA EXPLORACIÓN MOTRIZ EN EDUCACIÓN INFANTIL
}

Designing "settings" for motor exploration in early childhood education Orientações para a concepção de espaços para a exploração motora na educação infantil

\author{
Alfonso García Monge \\ Universidad de Valladolid. Telf.: +34983184071. Correo electrónico: \\ agmonge@mpc.uva.es \\ Francisco Abardía Colás \\ Universidad de Valladolid. Telf.: +34979108225. Correo electrónico: \\ abardia@mpc.uva.es
}

\section{Resumen}

Los materiales y espacios condicionan nuestra acción, de manera que se pueden diseñar escenarios para la práctica motora organizados en función de ciertas intencionalidades educativas. En este artículo mostramos algunas orientaciones para posibilitar la multiplicación de posibilidades de acción, así como la progresión en dificultad en las diferentes habilidades.

Palabras clave: motricidad, psicomotricidad, Educación Física, Educación Infantil, diseño de espacios de aprendizaje.

\begin{abstract}
Space and objects oriented the human action and we can design settings according to our pedagogical aims. In this article we try to show some orientations to enable the multiplication of possibilities of action as well as the progression in difficulty in the different abilities.

Keywords: motor behavior, Physical Education, Pre-shool Education, Learning Environments Design.

\section{Resumo}

Os materiais e espaços condicionam a nossa acção, de modo a que os cenários possam ser concebidos para a prática motora organizada de acordo com determinadas intencionalidades educativas. Neste artigo mostramos algumas orientações para tornar
\end{abstract}


possível a multiplicação de possibilidades de ação bem como a progressão em dificuldade nas diferentes habilidades.

Palavras-chave: habilidades motoras, psicomotricidade, Educação Física, Educação Infantil, desenho de espaços de aprendizagem.

\section{Introducción}

Partiendo de la idea de la importancia de los materiales y espacios en el desarrollo de los procesos de exploración del alumnado, presentamos algunas ideas para orientar el diseño de espacios que ayuden a multiplicar las habilidades motrices del alumnado, intentando respetar unos principios de progresión en la dificultad. Para ello, situamos nuestro trabajo respecto algunos conceptos teóricos básicos en los que nos apoyamos y mostramos algunos criterios básicos para organizar espacios que sugieran la motricidad y permitan una exploración del mismo por los niños y niñas.

\section{Algunos conceptos previos en los que se apoya esta propuesta}

Desde finales del siglo XIX, la pedagogía comenzó a verificar las relaciones de los entornos de aprendizaje con las respuestas de los aprendices. Por su sistematicidad y repercusión posterior, es de destacar el trabajo de María Montesori y la importancia que daba a los ambientes y materiales preparados por las "Guías": La educación no se adquiere escuchando palabras, sino por virtud de experiencias efectuadas en el ambiente. La función del maestro no es hablar, sino preparar y disponer una serie de motivos de actividad cultural en un ambiente especialmente preparado $(1986,19)$.

Durante el siglo XX, las teorías psicológicas irían confirmando estas influencias entre persona y contexto. Recordemos el enfoque socioconstructivista de Vigostsky, los planteamientos de la Teoría de Campo de Kurt Lewin o la Perspectiva Ecológica del Desarrollo Humano de Bronfenbrenner. El interés de estas relaciones entre persona y entorno llevaría a crear ramas de estudio como la Psicología Ambiental (al respecto se pueden ver los trabajos de Altman, 1975 o Stokols y Altman, 1987).

Es también a mediados del pasado siglo cuando desde la filosofía existencialista, Merleau Ponty introdujo el concepto de "intencionalidad motora" (1985) para referirse al proceso de las actividades intencionales de nuestro cuerpo en función de las características espaciales. Un concepto ratificado en la actualidad desde la neurociencia (Milner y Goodale, 1995) y que, de alguna manera, se encuentra en la base de las teorías 
contemporáneas del embodied cognition, el aprendizaje situado o el extended mind y otras más subjetivistas como la Enacción (Nöe, Varela-Maturana). Frente a posturas gestálticas como las de Köhler en las que el resultado de la actividad es resultado de fuerzas ajenas (los objetos orientarían la acción); Merleau Ponty (al igual que Piaget) cuestiona este sometimiento total a las condiciones del ambiente, incluyendo la experiencia y la acción del sujeto en ese proceso de interacción con el entorno. Así, el cuerpo, en su acción en el entorno (enacción) iría creando un mundo de significados (Di Paolo, 2013), de manera que, como señala Di Paolo, “conocer es participar”. Una idea que figuraba en la base de la propuesta Piagetiana sobre el desarrollo cognitivo, pero que el autor ginebrino circunscribiría al periodo sensorimotor (el conocimiento construido en la experiencia).

Siguiendo con la idea de la "intencionalidad motora", el entorno nos atraería a organizar nuestra acción de una forma determinada para que se dé una relación óptima. Según Gibson (1986), percibimos affordances, "ofrecimientos" u oportunidades para la acción. Éstas serían el resultado de la interacción entre las características del entorno y las del agente (en Norman, 1990, podemos encontrar un interesante desarrollo de las ideas de Gibson aplicadas al campo de la ergonomía). Así, ese affordance, sería el significado que un agente extraería de los objetos que le rodean y estaría condicionado por su posición y posibilidades de acción (por ejemplo, para un bebé tumbado en su cuna, una pelota le ofrece la posibilidad de ser chupada, pero unos meses más tarde, ya sentado en una silla puede usarlo como objeto para arrojar), la información contextual (e.g. Girardi, Lindemann y Bekkering, 2010), así como por los planes y circunstancias del agente (Pacherie, 2008). Como señala Jeannerod (1994), las características de los objetos activan adaptaciones motoras determinadas, siguiendo diferentes patrones en función del tiempo que es percibido el objeto (Tucker y Ellis, 2004), la preparación postural previa o la posición del agente respecto al objeto (Craighero, Fadiga, Rizzolatti y Umilta, 1999), o la congruencia de la acción con el objeto (e.g. Riddoch, Edwards, Humphreys, West y Heafield,1998; Tucker y Ellis,2001).

Como señalábamos arriba, la pedagogía lleva más de un siglo preocupada por crear ambientes que estimulen en los aprendices la exploración y el descubrimiento de las leyes que rigen el mundo físico y cultural. El trabajo de la doctora Montessori es pionero en la investigación de esos materiales que ayudan a través de la manipulación a comprender conceptos abstractos, advirtiendo a aquellos que no veían esa fuerza 
invisible de los ambientes: El error de la libertad del niño en la educación ha sido considerar una hipotética independencia del adulto sin la correspondiente preparación del ambiente. Esta preparación del ambiente es una ciencia educativa (Montessori, 1987, 294).

Señala Wilson (1996, 4) que esos ambientes de aprendizaje (learning environments), frente a los ambientes de enseñanza (instructional environments), permiten a los aprendices enfrentarse con espacios, dispositivos, o materiales que les permiten interpretar, experimentar, resolver problemas e interactuar con ellos y con otras personas.

En el ámbito de la educación física y la psicomotricidad, nuestra experiencia es deudora de las propuestas de Lapierre y Aucouturier y, más concretamente, de las de uno de sus discípulos en España: Marcelino Vaca. En la obra "los contrastes" (1974), Lapierre y Aucoutorurier comienzan a hablar de una fase de actividad motriz espontánea como "periodo de actividad libre, de creatividad motriz que precede al estudio de cada noción" (p.27) para que puedan expresar, a través del movimiento, las pulsiones y motivaciones inconscientes, descubrir intereses del alumnado y permitirles evolucionar hacia situaciones más complejas partiendo de sus necesidades. Para ello sugieren poner a disposición de los escolares objetos que induzcan la exploración motriz.

Partiendo de estas ideas, Vaca (e.g. 1997, 2000, 2005 y 2008) comenzará a organizar las estructuras de la lección de educación física con escolares de entre 3 y 6 años en unas fases que pasarán de la "Exploración" inicial (los niños y niñas exploran libremente en el gimnasio a partir de los espacios y materiales que el docente ha preparado previamente), en la que el docente hará un "Ensayo de tareas compartidas" (acompañamientos, refuerzos, diálogos no verbales a partir de las respuestas del alumnado), hacia fases finales de "Tareas compartidas" (en las que el docente va dotando de nuevos significados a las formas de hacer con los materiales y espacios propuestos). En esa línea, Abardía, Medina y Martín (1996) proponen una metodología guiada a través de lo que denominan "Recorridos Aventura" inspirándose en el diseño intencionado de los espacios para hacer surgir la motricidad de forma espontánea y natural. 
Serán estos trabajos de Vaca y Abardía los que orienten el planteamiento que aquí se expone.

3. ¿Qué criterios puede manejar el docente para organizar el espacio para posibilitar la exploración y expresividad del alumnado?

a) La seguridad: Consideramos que este es el aspecto básico sobre el que se construye el resto, aunque se trata de un concepto muy subjetivo dependiente del conocimiento de la acción por el ejecutante, el autoconcepto, la situación emocional, la cultura del contexto, etc.

En principio hablaríamos de la subjetividad del docente. Entendemos que se deben proponer tantos espacios como pueda controlar el adulto, es decir, tantos como le permitan estar tranquilo. Pero hay que tener en cuenta que un número muy reducido de espacios puede provocar ansiedad por una excesiva espera y ello puede llevar a salirse de la tarea, a descontrol, a presión en la ejecución, o una acción no reposada.

Podríamos hablar de otras normas de seguridad como:

- Colocar colchonetas en las zonas de recepción de los lugares de salto. Hay que tener en cuenta que las colchonetas gruesas (quitamiedos) pueden provocar abandono corporal y descontrol.

- Dejar clara la direccionalidad de los recorridos para evitar que los niños y niñas se puedan cruzar, chocar o ver frenada su acción por otros. Por ejemplo, las dianas de los lanzamientos deberían estar en el lugar opuesto al lugar en el que se hacen los equilibrios para evitar problemas.

- Evitar que se crucen la dirección de dos recorridos o que dos escenarios confluyan en un mismo lugar. Imaginemos un escenario de equilibrio que acaba en una colchoneta a la que se puede saltar desde otro lugar (espalderas, carrera desde un recorrido), supondría un lugar de conflictos.

- Alejar las zonas de cierta peligrosidad (trepa, saltos, equilibrios...) de otras de mayor movilidad (lanzamientos, carreras, franqueos). Se puede pegar a las paredes los escenarios relacionados con los saltos, equilibrios o trepas (a no ser que sólo se esté trabajando eso) y dejar el resto de la sala para otras acciones. 
- Tener previstos los posibles usos (affordances) de cada material y valorar si nos comprometemos a usarlo o no. Por ejemplo, el carácter dúctil de una cuerda puede llevar a usos como delimitar o hacer siluetas, obstáculo que franquear, objeto de unión (atar, arrastrar, colgarse...), objeto para golpear... Algunos de esos usos nos pueden resultar interesantes, pero habrá que prestar atención a usos problemáticos como la utilización de la cuerda como látigo, como cuerda de escalada, pero sin fijar con un nudo (a estas edades son pocos los niños o niñas que saben hacer nudos que permitan colgarse de la cuerda y, en todo caso, pueden quemarse las manos al intentar descender por una cuerda), o como medio para atar a otra persona (rodeando zonas sensibles como el cuello).

b) Provocar las habilidades deseadas: Una de las primeras cuestiones es decidir qué se busca con la lección y, en función de ello, elegir los materiales y construir los escenarios que inviten a ello.

Puede ser interesante construir los escenarios atendiendo a algunas cuestiones:

- Que diversifiquen los patrones de la habilidad dentro de la misma complejidad de ejecución. Imaginemos que hemos pensado en trabajar los lanzamientos, puede ser interesante ofertar situaciones de lanzamientos a dianas al ras del suelo (bolos, ladrillos...), otras elevadas (canastas, dianas en la pared), unas que supongan precisión, otras que supongan distancia, unas que precisen tiros directos, otras de tiros parabólicos, ...

- Que dentro de un mismo patrón ofrezcan diferentes niveles de dificultad para atender a la progresión y a las necesidades de diferentes escolares. Por ejemplo, si nos interesa la trepa por un banco inclinado apoyado en las espalderas, podríamos colocar bancos con diferentes grados de inclinación que den oportunidades a diferentes personas y les garanticen una progresión en esta habilidad.

- Que siempre que el material nos lo permita y el escenario vaya aglutinando los intereses del alumnado, multipliquemos el escenario para que pueda acoger la acción de más niños y niñas, evitando que éstos prolonguen sus tiempos de espera y así aumenten sus oportunidades de ejecución. 
- Que los escenarios que propongamos nos ofrezcan recorridos de progresión amplios que con pequeñas modificaciones permitan al escolar ir creciendo en la complejidad del reto al que se enfrenta.

- Que el escenario induzca la acción que queremos. La experiencia nos irá mostrando la affordance del diseño espacial que hayamos propuesto. Lo que para el docente supone un significado claro, puede que no lo sea tanto para el alumnado.

c) El equilibrio entre lo fijo y lo cambiante: Consideramos interesante que en la sala haya cosas fijas y otras que vayan cambiando paulatinamente. Ello supone un juego entre lo que somos (lo conocido, lo que nos da seguridad, lo que nos hace sentirnos partícipes de un contexto) y lo que podemos llegar a hacer (lo que nos reta, lo que cae dentro de nuestra zona de desarrollo próximo y nos atrae por esa proximidad) Es decir, el juego entre las estructuras que tenemos (asimilación) y las que vamos generando al enfrentarnos a situaciones novedosas (acomodación).

Al ofertar escenarios que prolongarán su permanencia en la sala durante el tiempo (varias semanas), se puede producir en el alumnado un efecto tranquilizador dado que no actuará con la premura que supone el hacer en un lugar pasajero y sabiendo que en aquel contexto hay lugares conocidos en los que puede sentirse seguro.

Por otra parte, las cosas que se prolongan en el tiempo permiten la progresión individualizada, respetando los diferentes tiempos y estilos de aprendizaje.

Lo cambiante es la invitación a la nueva exploración, a los nuevos caminos para la acción y el aprendizaje, al estímulo constante dentro de un microsistema en el que cada vez se va siendo capaz de hacer más cosas y ser más autónomo.

Este juego entre la seguridad y la inseguridad, lo conocido y lo novedoso asegura que más personas se verán incluidas y atendidas en el proyecto.

Cada lugar puede ser enriquecido por las propuestas del adulto: formas de lanzar, formas de equilibrarse... Pero, a partir de ahí, cuando un escenario va siendo dominado por el grupo, se pueden hacer pequeñas modificaciones que le den un nuevo aspecto, abran nuevos desafíos y despierten la curiosidad del alumnado por seguir retándose y descubriendo. 


\section{Parámetros básicos para construir escenarios. Ejemplos para las habilidades de salto y lanzamiento}

En el diseño de escenarios que sugieran la exploración motriz, para atender a las directrices anteriormente expuestas sobre seguridad, diversificación de patrones o progresión en la complejidad, seguimos un procedimiento analítico por el que descomponemos las habilidades en las variables que pueden afectar a su realización.

Imaginemos las habilidades de saltos. Podemos hacer un desglose de las variables que intervienen como el siguiente:

- Aproximación-batida: parado-desplazamiento, superficie estable-inestable, inclinación de la superficie, altura de la base de salida, propulsión (si la base es reactiva o no), posición del cuerpo, parte del cuerpo que impulsa, posibilidades perceptivas (plenas o limitadas, e.g. poniendo un parche en un ojo para provocar nuevas adaptaciones perceptivas en el cálculo tridimiensional), posibilidades motrices (plenas o limitadas, e.g. poniendo algún objeto en la cabeza que hay que evitar que se caiga durante el salto),...

- Fase aérea: ascendente-descendente, en altura o en longitud, superando un obstáculo (móvil-fijo), adaptando el cuerpo a un obstáculo, modificaciones en la posición del cuerpo (giros, agrupamientos, extensiones, agarres,...).

- Recepción: estabilidad-dureza-inclinación-amplitud-altura de la base, puntos de apoyo corporales, enlace con un nuevo impulso,...

Cuando hay que enlazar varios saltos seguidos (imaginemos una rayuela o un circuito con aros) aparecen otras variables que tienen que inciden en la dificultad perceptiva y coordinativa.

Algunas de esas variables hablan sobre condiciones del espacio en el que se desarrolla la acción (e.g. la posición de la superficie de batida con respecto a la de recepción) y que condicionarán el patrón a usar. Otras nos hablan de aspectos sobre las posiciones corporales o sus limitaciones perceptivas y no serán modificables a través de una organización del espacio durante la exploración libre, sino que necesitarán una consigna o sugerencia verbal del docente. 
Para construir los escenarios podemos ir haciendo combinaciones de las anteriores variables atendiendo a recomendaciones evolutivas genéricas como las de Wickstrom (1990):

- Dejarse caer desde poca altura (aumento progresivo de la altura)

- Salto vertical

- Salto en longitud

- Salto a franquear un obstáculo fijo

- Salto a subir en algún sitio

- Encadenamiento de saltos

- Salto a franquear obstáculos móviles

En el caso de las habilidades de lanzamiento, podríamos contemplar variables como las siguientes:

- Móvil: Tamaño, forma, peso, textura, sonoridad...

- Cómo se lanza: manejo directo-indirecto (implementos), parte del cuerpo, posición de partida, orientación con respecto a la diana, posibilidades perceptivas, tiempo de contacto con el móvil (golpeo),...

- $\quad$ Superficie de partida: estabilidad, altura,...

- Recorrido del móvil: directo-indirecto (hacer rebotar el móvil en algún lugar antes de llegar al objetivo), visible-no visible, libre-obligado (el móvil debe pasar en su fase aérea por alguna meta anterior a la diana final), trayectoria vertical ascendente-descendente, trayectoria recta (por una superficie, por el aire), trayectoria parabólica (tipo de parábola).

- Diana: tamaño, forma, altura, distancia, posición con respecto al plano del suelo, movilidad,...

Igualmente, observamos que hay variables que afectan al espacio de acción (objetos, materiales, dianas) y otras que tienen que ver con las posibilidades perceptivomotrices de la persona que realiza la acción. Las primeras podrán ser organizadas por el docente para posibilitar la exploración y las segundas requerirán de la sugerencia del adulto. 
Siguiendo a Wickstrom (1990) tenemos las siguientes guías como orientaciones generales para construir de forma progresiva los escenarios:

- Distancia cercana y trayectoria descendente (dejar caer)

- Trayectoria plana sobre el suelo o una superficie que induzca el recorrido del móvil (rodando por el suelo, por un banco, por un tubo cortado,...)

- Por el aire, pero con una trayectoria plana

- Por el suelo pero con un recorrido sinuoso, no llano

- Por el aire con una trayectoria parabólica (más dificultad en el cálculo del recorrido del móvil)

- Con trayectorias indirectas (botando o rebotando en algún sitio)

- Sobre dianas móviles

- Lanzamientos sin ver la diana directamente

- Lanzamientos sin ver la trayectoria pero sí la diana

\section{Los escenarios para el salto}

\subsection{Claridad del escenario}

Los escenarios de salto pueden tener varias partes que, al observarlas, deben percibirse como un todo. La superficie de aproximación o batida, el obstáculo posible y la zona de recepción deberían estar diseñadas de manera que al mirar al escenario se produzca una asociación entre ellas que sugiera el salto.

No conviene hacer las delimitaciones con objetos móviles (que se puedan llevar de allí) o que sugieran otras actividades (cuerdas, aros,...).

Los aros, sobre determinadas superficies lisas, pueden suponer un peligro dado que al pisar sobre ellos se puede uno resbalar. Con una gota de silicona seca que pongamos puede cambiar su adherencia.

Habría que diseñar el escenario de manera que se dé una direccionalidad clara a la acción. Por ejemplo, si colocamos una cuerda entre dos soportes sugiere el salto, pero en los dos sentidos, con lo cual se pueden provocar choques entre dos personas. Las 
colchonetas, como lugar de recepción, ayudan a orientar la acción dado que sugieren un lugar de llegada-caída.

Es interesante que nos aseguremos que el lugar al que se llega no está cerca de otro escenario de desplazamientos, saltos o lanzamientos que suponga un peligro para las personas que saltan.

Si realizamos varios niveles de dificultad conviene que queden un poco separados en el espacio para que no se usen de forma "transversal" y se provoque un choque. Por ejemplo, imagina dos plintos con diferentes alturas desde los que saltar a unas colchonetas. Si se colocan juntos puede sugerir saltar de uno a otro o de la colchoneta de recepción de uno a la del otro.

\subsection{Niveles de dificultad}

Al igual que en el caso de los lanzamientos, el cambio en la graduación de dificultad de alguna variable lleva al cambio de patrón a utilizar. Una mayor altura del obstáculo, una menor base de recepción, o un aumento en la longitud del salto pueden provocar el uso de un patrón motor diferente que haga que no se progrese sobre una acción concreta, sino que se cambie de acción.

Cada posible escenario tiene a su vez un largo recorrido de uso y progresión que viene determinado por las propuestas que el docente haga en él. Por ejemplo, el salto desde un banco al suelo se enriquece cuando el docente va dando nuevos caminos de uso: sin apoyar las manos al caer, saltar con las manos unidas, saltar lateral, hacia atrás, recibiendo un objeto,... Esas consignas irán en función de las posibilidades de cada uno. Es decir, el maestro o maestra pueden ir retando a cada niño o niña a realizar aquello para lo que vayan estando preparados. Es en ese momento en el que aparecerán los criterios de realización y las normas de seguridad de cada técnica (dónde mirar, cómo impulsar, cómo equilibrarse, cómo recepcionar,...).

\subsection{La seguridad, el logro}

Desde que comenzamos a ganar en verticalidad, se va produciendo en nosotros un placer por ser más y ver el mundo desde otra perspectiva $y$, de forma paralela, un miedo a la caída. Según sea la actitud de la familia, el miedo a la caída puede llegar a desaparecer jugándolo, integrando ésta como parte del placer del salto (Aucouturier, 2004). 
Frente al esfuerzo de incorporarse aparece el placer del abandono en la caída (hablamos de superficies mullidas). Señala al respecto Aucouturier (2004, 84): Al caer con placer, el niño muestra a sus padres su seguridad en sí mismo [...] El placer de la caída permite la afirmación de sí frente a los padres y es la prueba de una separación bien asumida.

Hay, por tanto, un factor emocional en estas actividades que provocan la crispación y rechazo de aquellos niños y niñas más inseguros. Los escenarios deberían permitir el disfrute con las sensaciones básicas de caída. Para ello, hay que asegurar la posibilidad de que cada uno elija la altura de salto que desee y buscar escenarios en los que se dé un espacio de recepción agradable.

Por otra parte, el salto tiene un componente de autoafirmación. Es, por ello, interesante que el alumnado vaya teniendo oportunidades y referencias de sus logros y progresiones. Desde dónde o cuánto saltaba antes y lo que va consiguiendo con el trabajo.

Hay diferentes fuentes que pueden retroalimentar las ganas de volver a saltar: las hedonistas derivadas del placer de caer en lugares mullidos (colchonetas quitamiedos, cojines, piscinas de balones o de espuma,...); el placer de verse causante de algún efecto (saltar tocando algún móvil colgado del techo o de unas escaleras horizontales este placer puede verse incrementado si el móvil emite sonido-); y el placer de autoafirmarse viendo que se logra superar nuevos retos y que se va logrando superar niveles de dificultad (superar diferentes alturas, llegar más lejos, saltar desde más alto,...).

\subsection{En qué incidir}

A los 6 años, los niños que han tenido ocasión de enfrentarse a diferentes situaciones gratificantes de salto, son capaces de saltar desde alturas considerables (desde lo alto de las espalderas, desde un plinto,...). Su posibilidad de saltar es mayor que el tono que tienen para amortiguar la inercia y se pueden ver movimientos de rebote de sus diferentes articulaciones. Esto conlleva un peligro de lesiones o pequeños microtraumatismos.

Pensamos que llegado a un punto, la habilidad habría que hacerla crecer no hacia lo físico-motriz (saltar más alto, desde más alto, más lejos,...un camino que puede llevar a lesiones), sino hacia lo perceptivomotriz y neuromotriz (saltar más coordinado, 
con más precisión, ante situaciones de mayor complejidad perceptiva, de más formas,...). Es el caso de los escenarios de encadenamiento de saltos. Su sencillez de diseño choca con la complejidad coordinativa y perceptivomotriz que pueden suponer (adaptaciones continuas que exigen medir la fuerza de impulsos, el control de las recepciones, la adaptación a fases aéreas cambiantes, el control de aceleraciones y deceleraciones...).
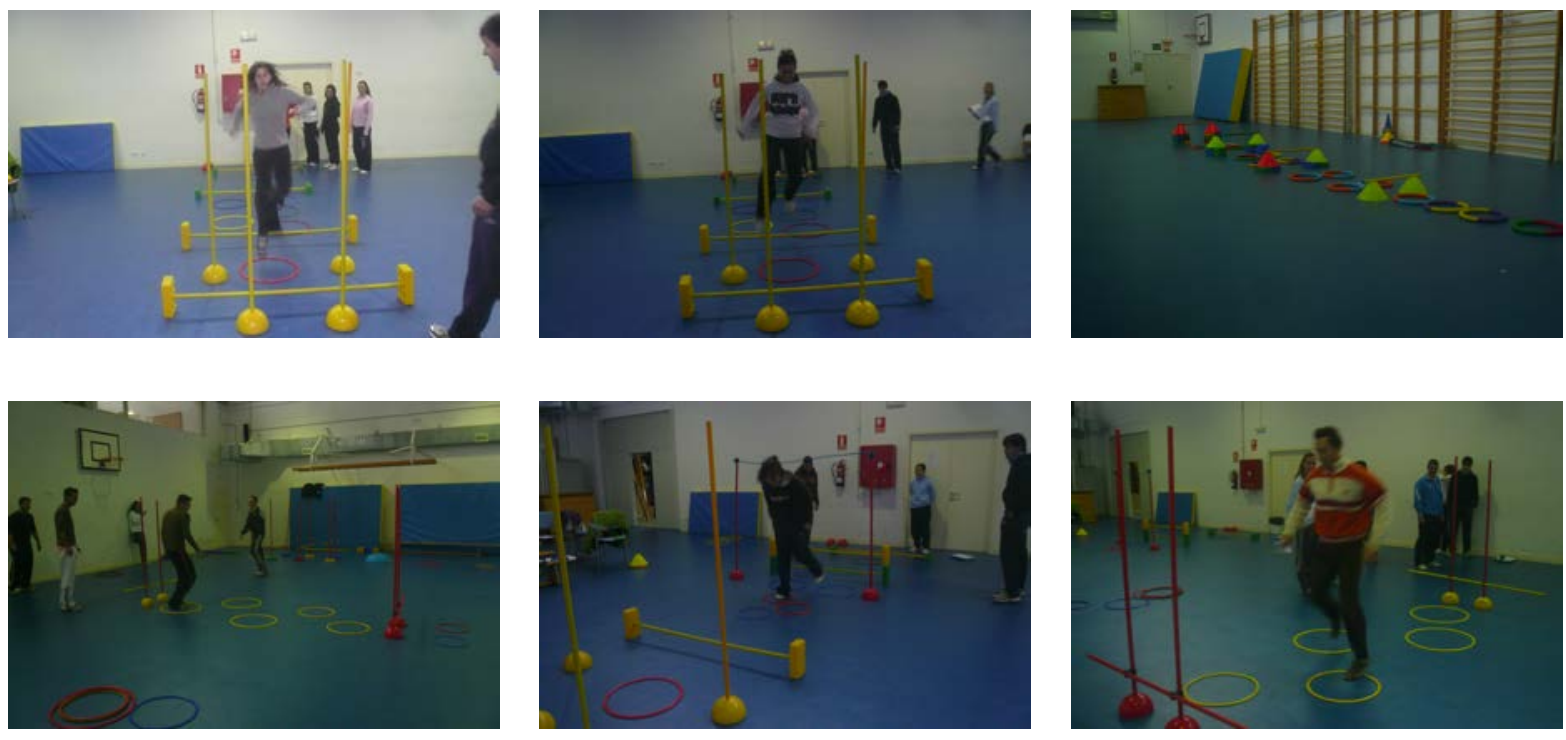

Por otra parte, los escenarios que tienen un componente fisicomotriz muy marcado se consumen pronto. Por ejemplo, si colocamos unos globos colgados del techo o de las escaleras horizontales, los niños y niñas intentarán tocarlos unas cuantas veces, si lo logran abandonarán, y si no lo logran también. Por mucho que se esfuercen intuyen que hasta que no crezcan más o tengan más potencia de piernas no podrán conseguirlo.

\subsection{Detalles sobre la motricidad}

En la foto 1 podemos observar un tipo de reequilibración de brazos típico de estas edades (3-5 años) 


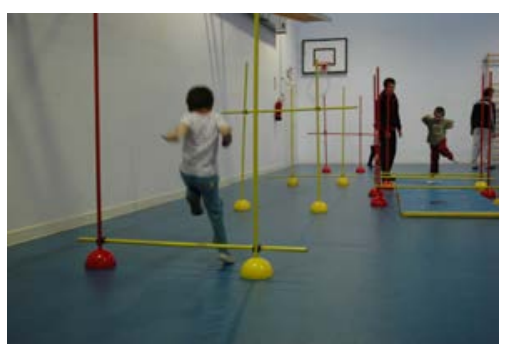

Foto 1

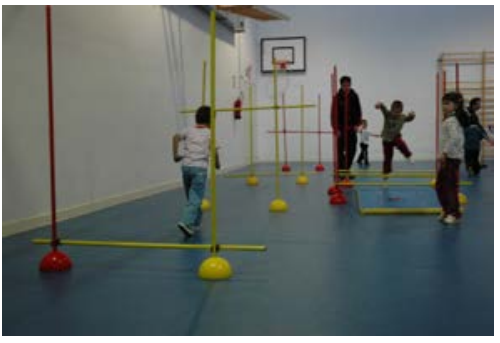

foto 2

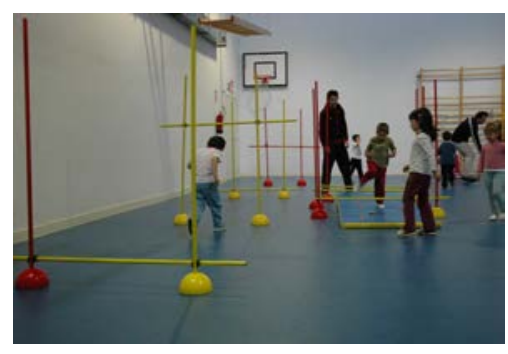

foto 3

Los brazos realizan una acción inversa a la dirección del movimiento, como de freno a éste. Apreciamos también el exceso de fase aérea (ver pierna izquierda en la foto 1 y ver el niño de la derecha en la foto 2) que nos indica un desajuste perceptivo, que provoca que el movimiento sea poco adaptado al entorno.

Aún así, ese tipo de salto en un franqueo de un obstáculo sería más evolucionado que, por ejemplo, el que aparece en estas imágenes.

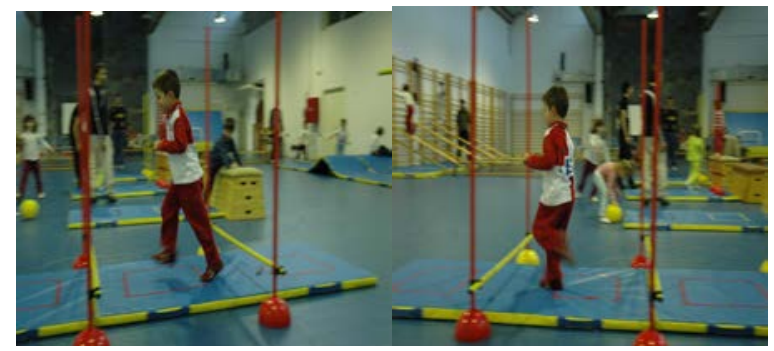

Se caracterizaría por una velocidad baja, sin fase aérea, proximidad máxima al obstáculo y rigidez en las articulaciones (rodilla, cadera, tobillo).
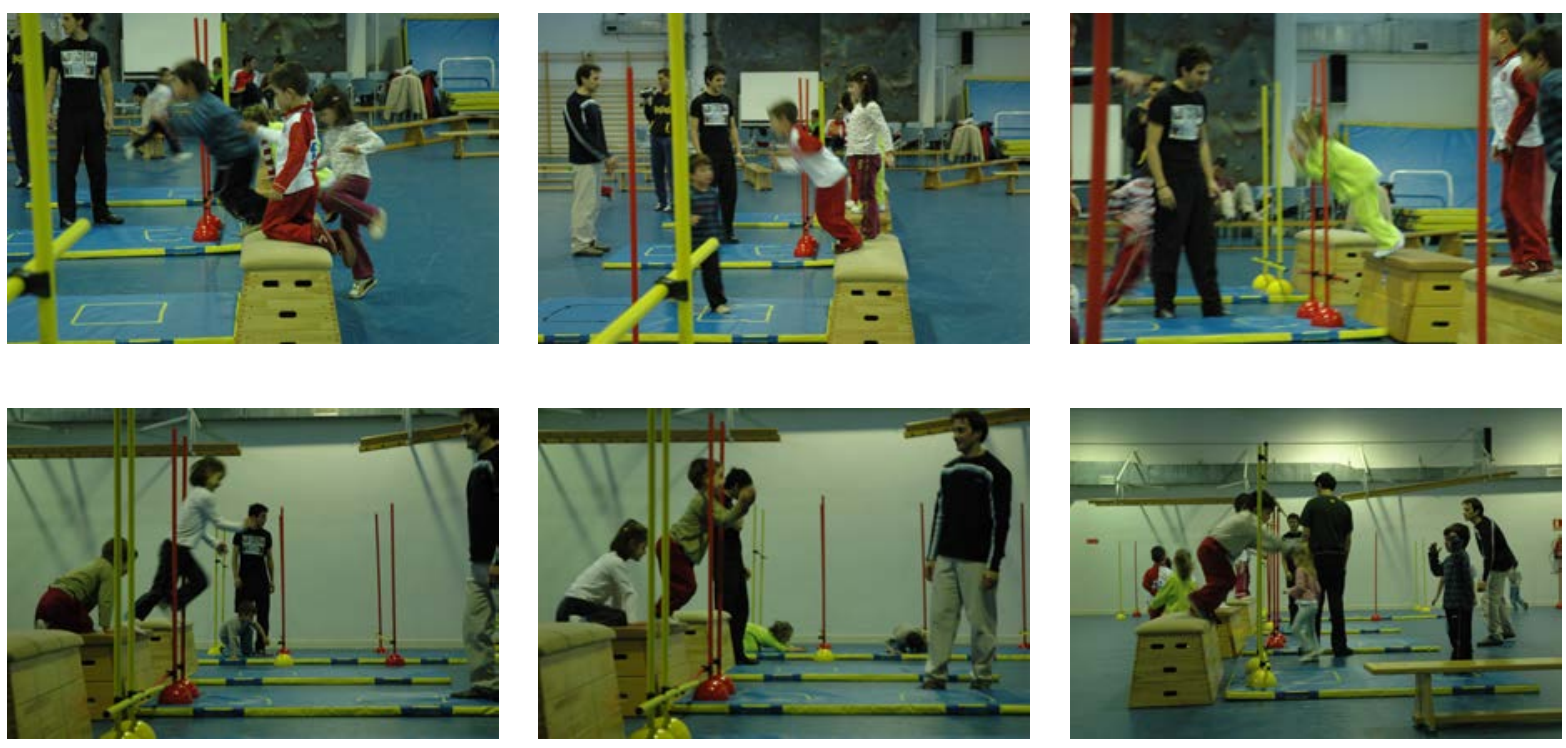

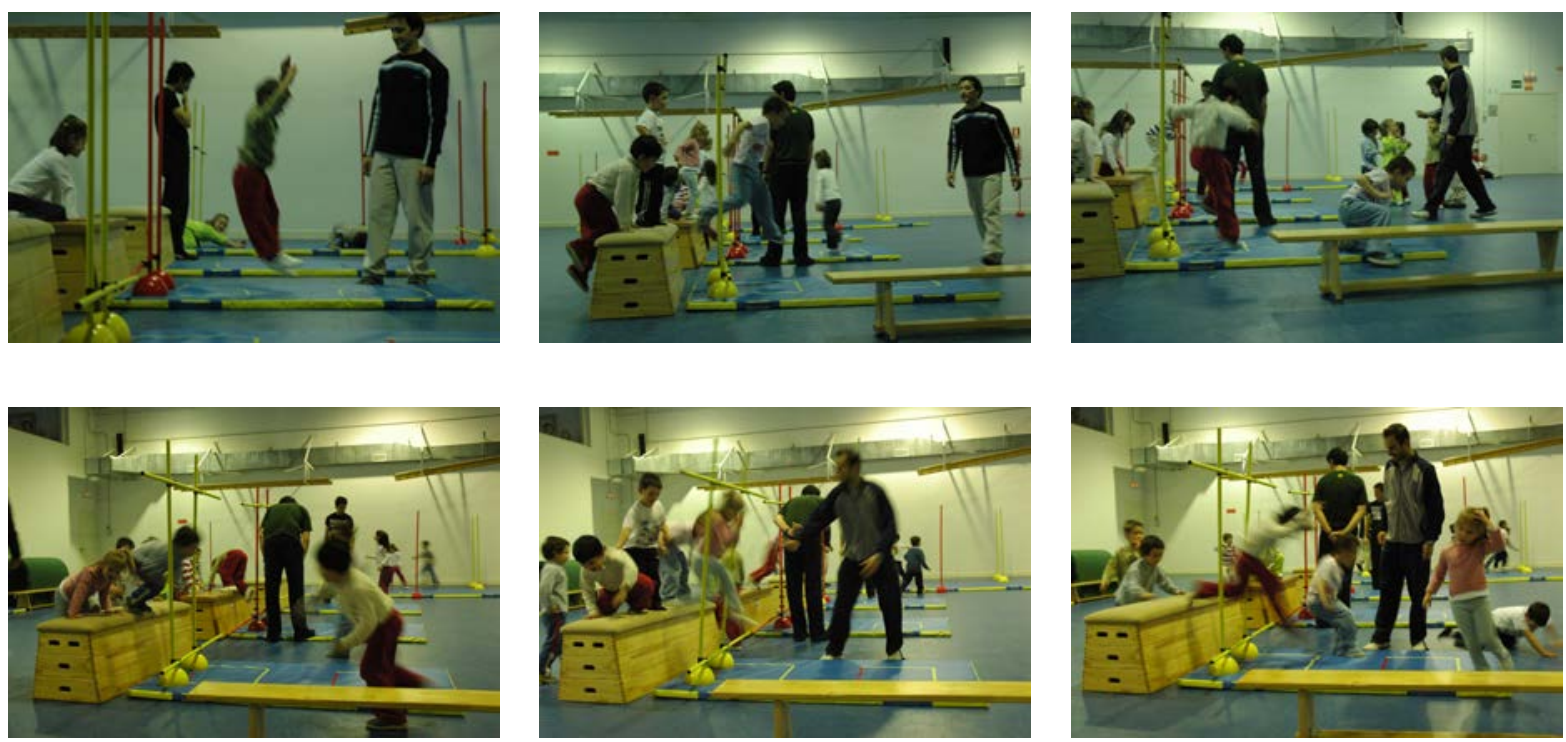

Estas fotos nos permiten ver formas personales de afrontar un salto. También nos muestran cómo el diseño del escenario puede condicionar la forma de saltar obligando a los practicantes a buscar nuevas adaptaciones corporales para resolver el reto.
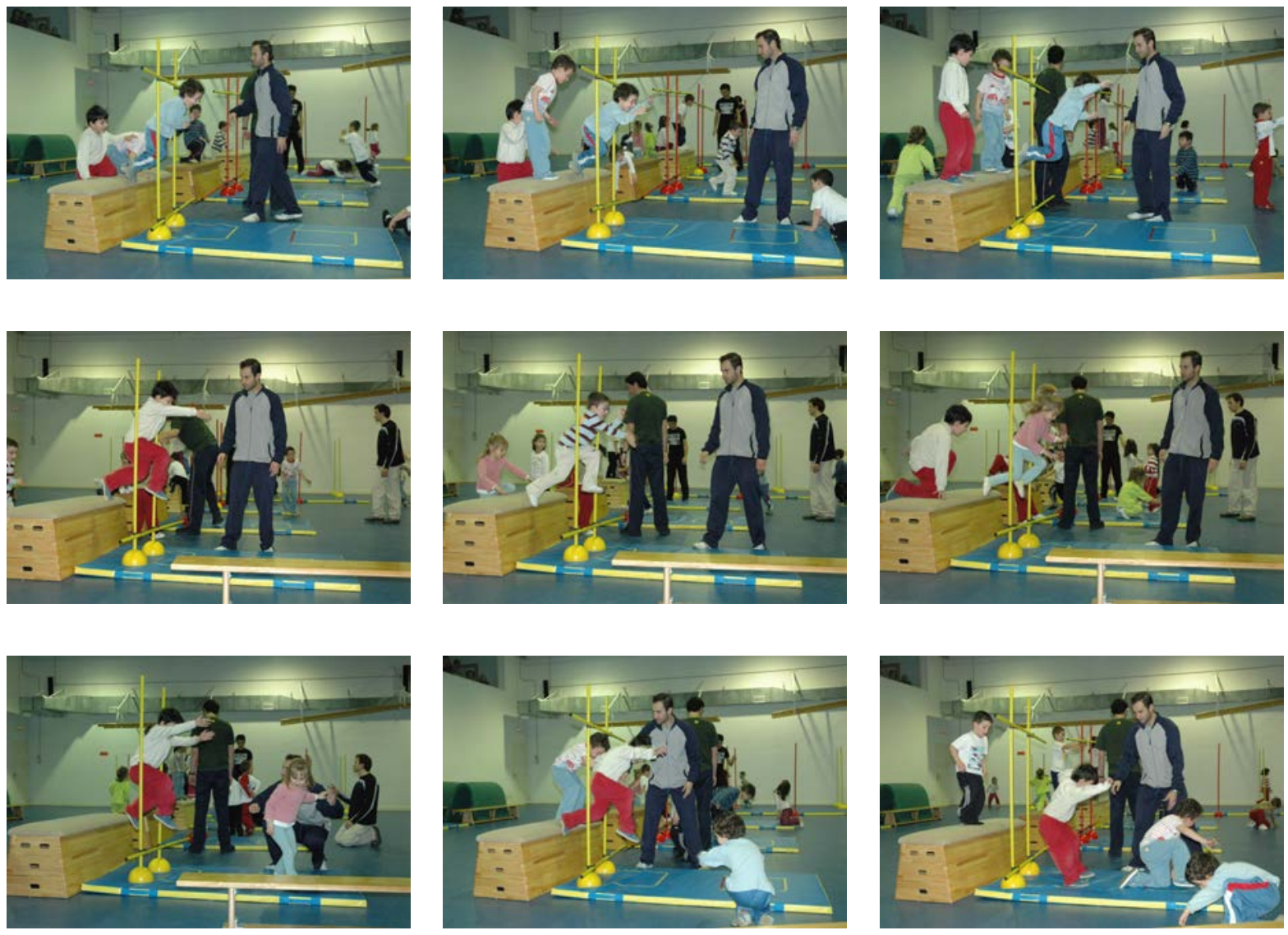

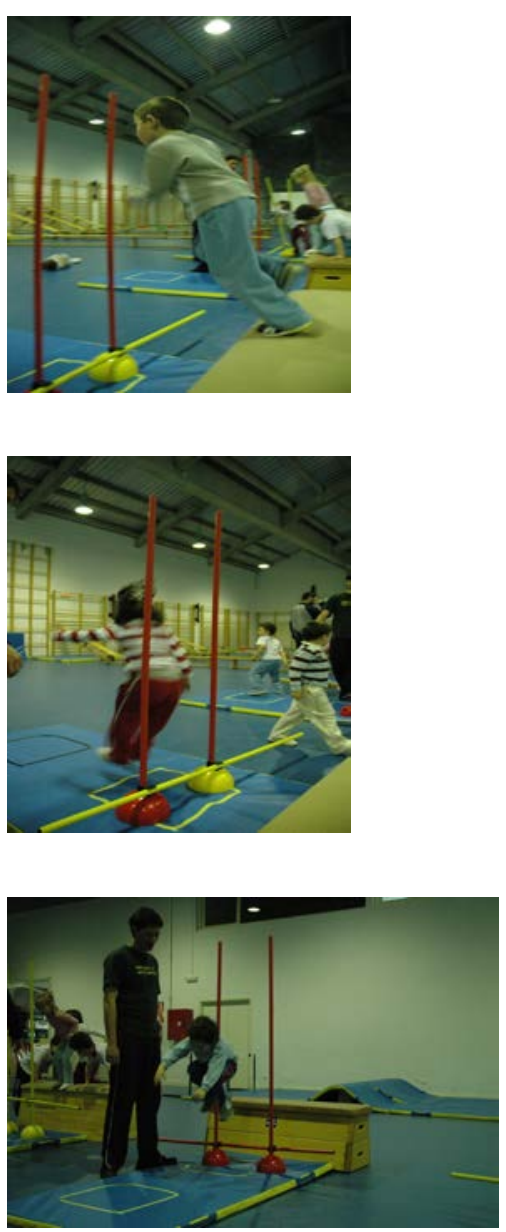
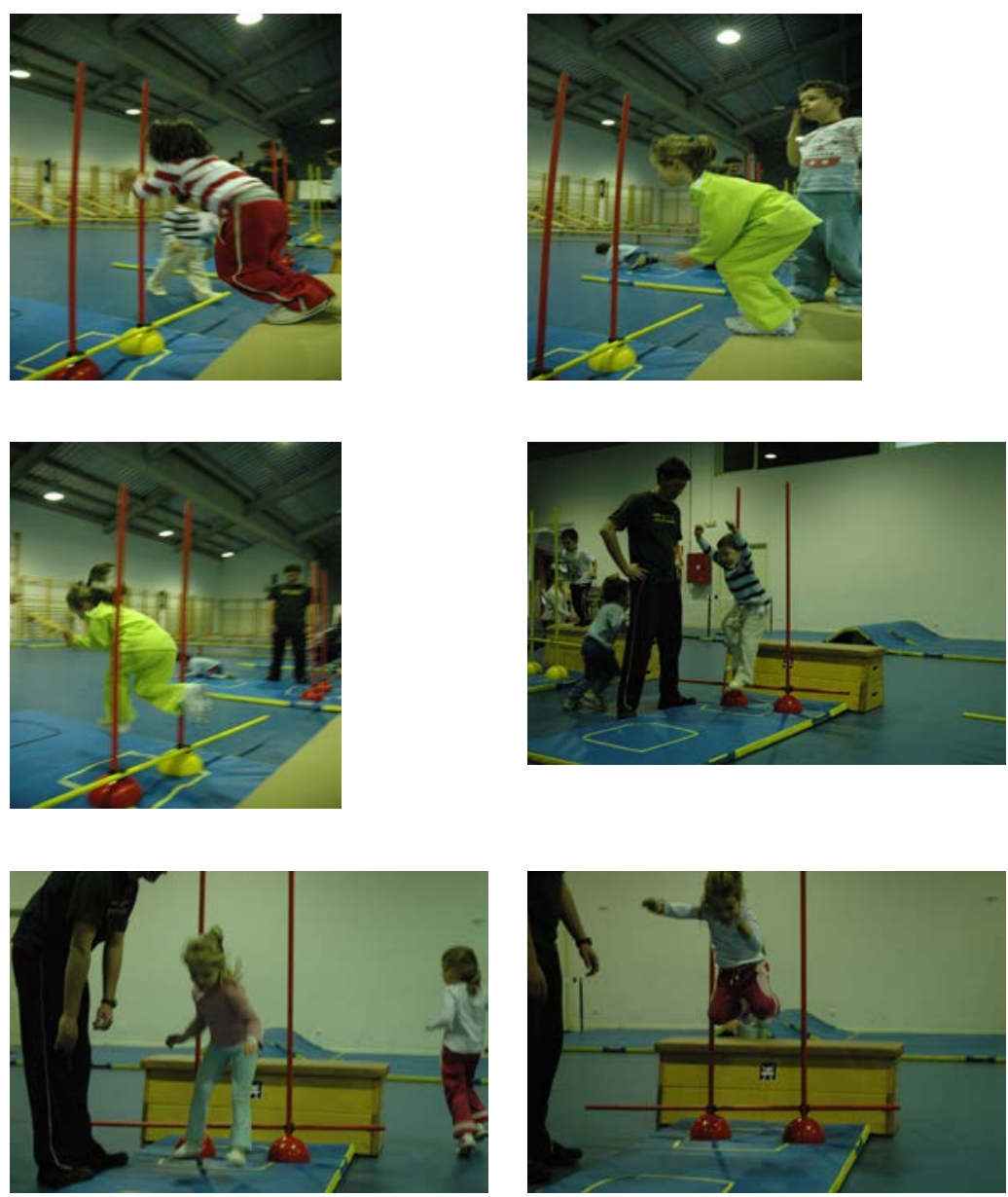

Estos escenarios se construyen posibilitando diferentes niveles de dificultad (altura del plinto, anchura entre listones, altura del listón inferior o distancia marcada en la colchoneta de recepción) y el propio escenario funciona como un sistema de control de los resultados en la medida que les da información sobre si logran cruzar los listones o no o hasta dónde son capaces de llegar (cuadrados marcados en las colchonetas).

\section{Los escenarios para lanzamientos}

\subsection{Claridad del escenario}

Cuando la persona se acerca a uno de estos escenarios puede que no perciba las relaciones entre los diferentes materiales que componen el mismo y, por tanto, no le sugieran el tipo de acción imaginada por el docente. Al percibir es interesante que se pueda establecer una relación entre los diferentes materiales que componen el escenario. Que se asocien unos a otros. A ello colaboran los colores para la demarcación de la zona de lanzamiento, el móvil y la diana (e.g. si podemos, colocaremos un balón rojo, cerca de una diana roja y una línea o círculo rojo que delimite el lugar desde el que lanzar). 
En ese sentido, es interesante que los colores estén asociados a los niveles de dificultad y se mantengan (si es posible) de unos escenarios a otros (e.g. amarillo nivel inicial, verde nivel medio, rojo máxima dificultad).

Hay objetos que puede que no se asocien al escenario y, por sí solos, sugieran otras acciones. Imaginemos que dejamos cerca de la diana un pañuelo imaginando que lo van a usar para taparse los ojos; lo más seguro es que lo utilicen para otra cosa. Otro ejemplo: imaginemos que colocamos un monopatín para dar dificultad a la superficie desde la que se lanza, el monopatín sugeriría por sí solo mucho más que el resto del escenario, al percibirlo el niño se dejaría llevar por ese objeto y se desentendería del resto.

\subsection{Niveles de dificultad}

Ya hemos señalado que, en ocasiones, al intentar buscar niveles de dificultad diferentes, cambiamos de patrón de realización. Por ejemplo, si colocamos una diana a diferentes alturas, sin querer estamos obligando a utilizar diferentes patrones de lanzamiento, con lo cual ya no se produce una evolución o mejora en uno de ellos, sino que se vuelve a empezar de cero con la siguiente diana.

Lo mismo ocurriría con balones de diferente tamaño o peso. Hay una progresión en el tamaño (a una misma dimensión de la diana): por ejemplo, podremos ir cambiando un móvil de tamaño siempre que todos ellos se puedan coger con una mano. En el momento que el alumno tenga que utilizar dos manos, habremos cambiado a otro patrón, y, por tanto, ya no habrá progresión de dificultad dentro del patrón inicial.

\subsection{Invitación a la acción del escenario}

El lanzamiento surge como un interés por proyectarse en el espacio e influir sobre él (Lapierre y Aucouturier, 1985). Al no poder desplazarse mucho, el niño ve que puede influir sobre el entorno mediante objetos intermediarios.

Es interesante, por tanto, trabajar a partir de esta idea del efecto que se causa en el espacio. Si la diana produce ruido o movimiento, el niño recibe una retroalimentación perceptiva (sonora, visual) que puede incrementar su fijación en esa tarea.

Con cascabeles en las dianas, o dianas inestables que se derrumben se incrementa la sensación de "poder", de "haber sido causante de...". El problema que tiene este tipo de dianas es la necesidad de volver a colocarlas y, a no ser que los 
escolares tengan bien asumida la normativa de funcionamiento, nos podemos encontrar con que tras tirar los bolos, bloques, aros, etc. nadie se preocupa de volverlos a colocar, con lo cual el escenario queda inservible. Por ello, tras esa invitación inicial al lanzamiento "destructivo" tendríamos que ser capaces de "engancharles" en el reto de medir su competencia e intentar superarse.

Hay escenarios cuyo atractivo está en su diseño o novedad. Imaginemos un banco sueco enganchado en las espalderas a modo de tobogán, en la parte baja colocamos un cubo, y dejamos una pelota pequeña junto a él. La sugerencia de dejar caer la pelota rodando para que entre en el cubo es casi inmediata. En este caso es posible que el niño o niña se vea motivado a realizar acciones que proyecten sus deseos, la pelota se convierte en una extensión de él y su deseo de deslizarse por el tobogán tal y como ocurre en los juegos simbólicos con muñecos o en juegos de proyección como las cometas (Pelegrín, 1986).

\subsection{Detalles sobre la motricidad}

Veamos con las siguientes imágenes algunos detalles sobre la habilidad a estas edades.

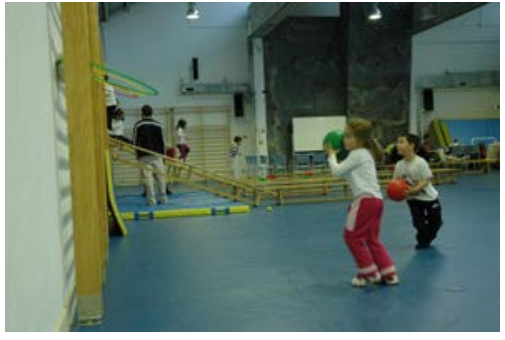

Foto 1

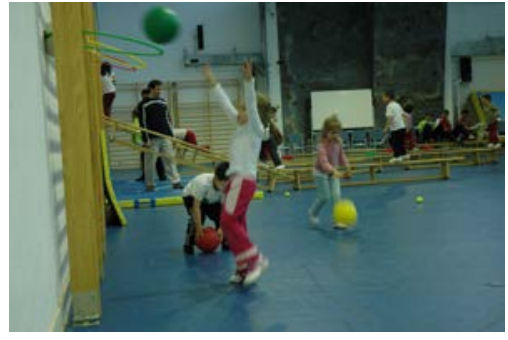

Foto 2

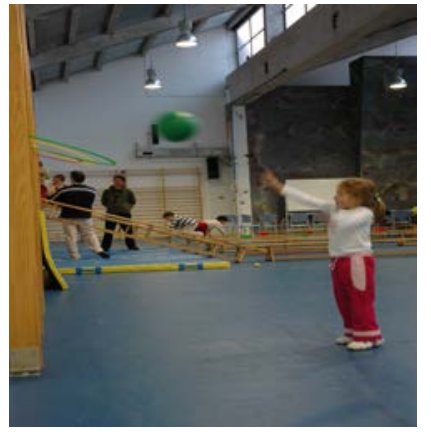

Foto 3

Las fotos 1 y 2 nos muestran una secuencia de lanzamiento a un aro. Es un patrón bastante avanzado para la edad de esta niña (4 años) como nos lo indican su distancia de la diana y la flexión de piernas en la fase inicial, pero mantiene detalles propios de la edad como acercarse a la diana o implicar todas las partes del cuerpo como si fuera una descarga eléctrica (extensión violenta de brazos y piernas). Sin embargo, las readaptaciones se producen rápidamente, el cuerpo reinterpreta rápido los datos de lo ocurrido y formula una nueva propuesta más eficaz como vemos en la foto 3. Entre el lanzamiento de las fotos 1-2 y el lanzamiento de la foto 3 sólo ha transcurrido un intento y ya podemos ver un patrón que correspondería a una niña de 6- 
7 años. Ello nos habla de que la práctica continuada y las indicaciones con las que el docente puede ayudar contribuyen a que los niños y niñas alcancen antes los patrones que están dentro de su zona de desarrollo próximo. No decimos que esto sea el objetivo, pensamos que a estas edades los escolares deben enfrentarse con muchas situaciones motrices, experimentar, dar soluciones divergentes e ir construyendo poco a poco los patrones que les resulten más eficaces.
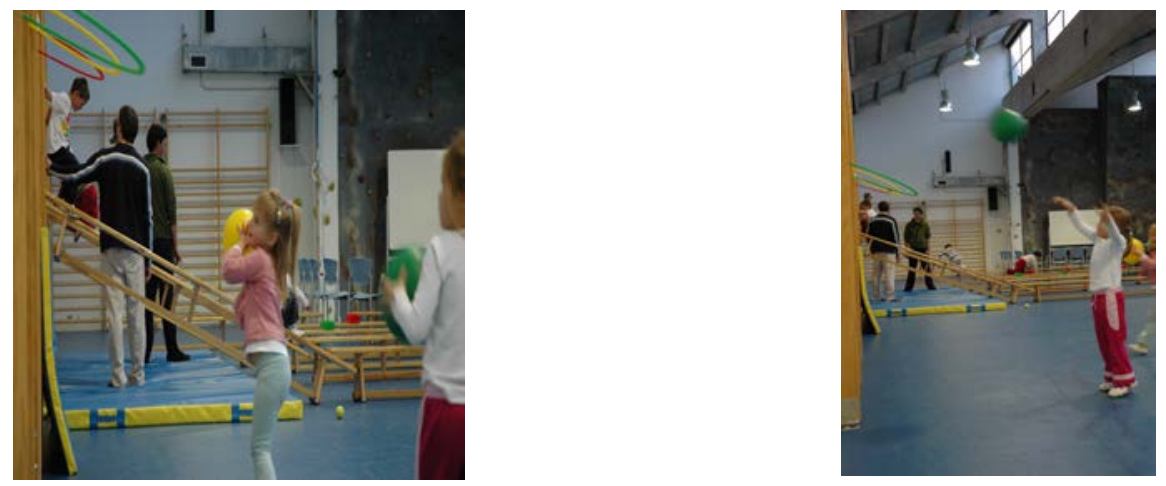

\section{Breves conclusiones}

Criterios de seguridad; provocar las habilidades deseadas (diversificando patrones, ofreciendo diferentes niveles de dificultad, multiplicando los escenarios, ofreciendo progresiones, atendiendo a que el escenario o material sugiera la acción deseada); y equilibrando lo que se repite con lo novedoso.

En la planificación de escenarios se sigue un procedimiento de descomposición analítica de cada habilidad para asegurarnos de que a través de los escenarios vamos solicitando diferentes patrones motrices.

Al construir los escenarios debemos asegurarnos de que si el escenario tiene varias partes (e.g. un balón, unas señales que indiquen diferentes distancias hasta la diana y una diana), perceptivamente se sugiera una relación entre las diferentes partes (e.g., si se puede, asociar objetos mediante colores similares -marcas de la zona de lanzamiento, pelota y diana del mismo color-). Igualmente, debemos asegurar que el escenario sugiere una trayectoria de uso y que esta trayectoria no es cortada por las de otros escenarios para evitar choques y conflictos. Otra idea básica es que el objeto no genere significados afectivos o simbólicos más fuertes que la habilidad esperada (muñecos, peluches, pelotas decoradas con personajes...). 
Todo escenario se puede ver enriquecido por las propuestas que el docente sugiere a partir de él, así como con los criterios de realización que éste pueda dar como feedback a cada participante.

Entendemos que es importante que el docente de oportunidades de que cada escolar sienta el éxito en la tarea, trabajando según sus posibilidades y facilitando instrumentos para que sea consciente de su progreso personal (los propios escenarios pueden estar construidos para servir como feedback).

Hay que tener en cuenta que las progresiones son muy personales. Cada niño o niña irá probando diferentes patrones y llegará a realizaciones con leves matices personales determinados por su mecánica articular, coordinación, experiencias previas, etc. El interés de utilizar ciertos criterios para el diseño de escenarios estaría en que nos permite tener algún "mapa" con el que orientarnos en la progresión y da propuesta de diversidad de experiencias.

\section{Referencias bibliográficas}

Abardía, F.; Medina, D y Martín, M.A. (1996). Desde la habilidad motriz básica hacia la acrobacia. Propuesta para elaborar recursos en E.F. basada en la reflexión de la práctica en grupo. Ed. Asociación Cultural: Cuerpo, Educación y Motricidad. Palencia.

Altman, I. (1975). The environment and social behavior. CA: Wadsworth.

Aucouturier, B. (2004). Los fantasmas de acción y la práctica psicomotriz. Graó, Barcelona.

Craighero, L., Fadiga, L., Rizzolatti, G., \& Umilta, C. (1999). Action for perception: a motor-visual attentional effect. Journal of Experimental Psychology: Human Perception and Performance, 25, 1673- 1692.

Di Paolo, E. (2013). El enactivismo y la naturalización de la mente. En D. Chico, \& M. Bedia. Nueva ciencia cognitiva: Hacia una teoría integral de la mente. Madrid: Plaza y $\quad$ Valdes Editores. https://ezequieldipaolo.files.wordpress.com/2011/10/enactivismo_e2.pdf

Girardi, G., Lindemann, O., \& Bekkering, H. (2010). Context effects on the processing of action-relevant object features. Journal of Experimental Psychology: Human Perception and Performance, 36, 330-340. 
Gibson, J. J. (1986). The Ecological Approach to Visual Perception. Nueva York: Psychology Press.

Jeannerod, M. (1994). The representing brain: neural correlates of motor intention and imagery. Behavioral and Brain Sciences, 17 (2), 187-245. http://users.abo.fi/jtuomain/EMCL/articles/additional/Jeannerod1994.pdf

Köhler, W. (1929/1967). Psicología de la configuración. Madrid: Morata.

Lapierre, A. y Aucouturier, B. (1985). Simbología del movimiento. Madrid: Editorial Científico-Médica.

Merleau-Ponty, M. (1945/1985). Fenomenología de la percepción. Barcelona: PlanetaAgostini.

Milner, A.David y Goodale, Melvyn A. (1995). The Visual Brain in Action. Oxford: Oxford University Press.

Montessori, M. (1986): La mente absorbente. Ed. Diana: México.

Montesori, M. (1987): El niño, el secreto de la infancia. Diana, México.

Norman, D. (1990). Psicología de los objetos cotidianos. Madrid: Nerea

Pacherie, E. (2008). The phenomenology of action: A conceptual framework. Cognition, 107 (1), pp.179-217.

Pelegrín, A. (1986). Cada cual atienda su juego: de tradición oral y literatura. Madrid: Cincel.

Riddoch, M. J., Edwards, M. G., Humphreys, G. W., West, R., \& Heafield, T. (1998). Visual affordances direct action: neuropsychological evidence from manual interference. Cognitive Neuropsychology, 15, 645-683.

Stokols, D. y Altman, I. (1987). Handbook of Environmental Psychology: vol 1. New York: Wiley.

Tucker, M., \& Ellis, R. (2001). The potentiation of grasp types during visual object categorization. Visual Cognition, 8, 769-800.

Tucker, M. y Ellis, R. (2004). Action priming by briefly presented objects. Acta Psychologica, 116, 185-203. 
Vaca, M. (1997). Tratamiento Pedagógico de lo Corporal en Educación Infantil: Construir el significado educativo de una práctica desde la interpretación y explicación compartida de lo ocurrido. Revista Electrónica Interuniversitaria de Formación del Profesorado, 1, http://www.uva.es/aufop/publica/actas/viii/edinfant.htm

Vaca, M. (2000). Reflexiones en torno a las posibilidades educativas del Tratamiento Pedagógico de lo Corporal en el Segundo Ciclo de Educación Infantil. Revista Interuniversitaria de Formación del Profesorado, 37, 103-120.

Vaca, M. (2005). El cuerpo y la motricidad en Educación Infantil. El análisis de la práctica como base de la elaboración de un proyecto para el tratamiento del ámbito corporal en Educación Infantil. Revista Iberoamericana de Educación, $39,207-224$.

Vaca, M. y Varela, M.S. (2008). Motricidad y Aprendizaje. El Tratamiento Pedagógico del Ámbito Corporal (3-6). Editorial Graó: Barcelona.

Wickstrom, R. (1990). Patrones motores básicos. Madrid: Alianza.

Wilson, Brent G. (1994) Constructivist Learning Environments: Case Studies in Instructional Design. Educational Technology Publications: New Jersey. 